\title{
A. F. MONnA \\ Rapport sur la théorie des espaces linéaires topologiques sur un corps value non-archimédien
}

Mémoires de la S. M. F., tome 39-40 (1974), p. 255-278

<http://www.numdam.org/item?id=MSMF_1974_39-40_255_0>

CC Mémoires de la S. M. F., 1974, tous droits réservés.

L'accès aux archives de la revue « Mémoires de la S. M. F. » (http://smf. emath.fr/Publications/Memoires/Presentation.html) implique l'accord avec les conditions générales d'utilisation (http://www.numdam.org/conditions). Toute utilisation commerciale ou impression systématique est constitutive d'une infraction pénale. Toute copie ou impression de ce fichier doit contenir la présente mention de copyright.

\section{Numdam}


Table Ronde Anal. non archim. (1972, Paris)

Bull. Soc. math. France,

Mémoire 39-40, 1974, p. 255-278

RAPPORT SUR LA THEORIE DES ESPACES LINEAIRES TOPOLOGIQUES

SUR UN CORPS VALUE NON-ARCHIMEDIEN

\author{
A. F. MONNA
}

I. Histoire. Je rappelle que la théorie des valuations non-archimédiennes fut commencée par K. Hensel en 1907 ; en particulier il introduit les nombres p-adiques. L'étude fut continuée par Kürschak et Ostrowski. Dès le début ces mathématiciens ont étudié l'analyse dans les corps munis d'une valuation non-archimédienne, mais c'était ce que j'appelle l'analyse classique : la théorie des séries, convergence, les séries de Taylor, etc. Cette étude fut continuée par Schöbe en 1930 ; il a étudié la théorie des fonctions au sens classique. Cependant, je ne veux pas rapporter sur ces développements. Je traiterai l'analyse linéaire, c'est-à-dire la théorie des espaces de Banach et les espaces localement convexes sur un corps muni d'une valuation nonarchimédienne.

Il faut remarquer qu'il y a des résultats en analyse classique $n$. a. qu'on a obtenus avec les moyens de l'analyse linéaire. Je rappelle par exemple le beau théorème de $M$. van der Put exprimant que dans la théorie des équations différentielles en analyse $n$. a. le rôle des constantes en analyse réelle est remplacé pour ainsi dire par les fonctions dont les dérivées sont partout nulles - ce qui, en analyse n. a., n'implique pas qu'une telle fonction est identiquement égale à zéro comme conséquence du fait qu'un corps valué n. a. est totalement discontinu (voir $[14],[16])$.

La théorie des espaces de Banach $\mathrm{n}$. a. sur un corps $\mathrm{K}$ mini d'une valuation n. a. commençait plus tard.

Environs 1940 j'ai commencé l'étude systematique des espaces de Banach n. a., plusieurs années sans beaucoup de répercussions. J'avais obtenu une caractérisation d'une certaine classe d'espaces au moyen de séries que j'ai appelées séries orthogonales et j'avais démontré le théorème de Hahn-Banach sous quelques conditions spéciales. En 1952 Ingìeton (précédé par un travail de I.S. Cohen en 1948) réussit 
dans un article fondamental à établir le théorème de Hahn-Banach au cas non-archimédien. Il introduisait la notion d'"espace sphériquement complet" qui fut plus tard de première importance.

En 1958 j'ai đéfini la notion d'ensemble convexe dans un espace linéaire sur $K$ - ce qui exigeait une nouvelle définition faute d'un ordre sur $K$ - conduisant aux espaces localement convexes sur $\mathrm{K}$. Cela marquait une seconde étape dans le développement. C'était le début de plusieurs articles dans le domaine de la théorie des espaces linéaires topologiques sur un corps valué n. a. -théorie de la dualité, théorèmes structurelles, intégration - d'abord par plusieurs autres mathématiciens hollandais (Monna, van der Put, van Rooy, Schikhof, Springer, van Tiel). Mais bientôt des articles sur divers domaines de l'analyse non-archimédienne furent publiés dans un cercle international : en France Mme Amice, Gruson, Serre, en Allemagne l'école de Grauert et Remmert sur la théorie des fonctions analytiques, en U.S.A. Ellis, en India Raghunatan, Rangan, Rangachari, en Belgique Mme De Grande-De Kimpe, sans prétendre cependant que cette liste soit complète.

Je crois qu'on peut dire que dans les dernières années une troisième étape est en train de développement, à savoir la théorie des algébres de Banach non-archimédiennes, liée à l'analyse harmonique et la représentation de groupes, cependant sans que le développement de la théorie des espaces de Banach ait cessé.

Cela est la grande ligne du développement. Pour une bibliographie complétée jusqu'à 1970 voir [1]. Sans prétention d'être complet j'ai ajouté dans la bibliographie quelques travaux, parus après que la bibliographie dans ce livre fut fermée.

II. Méthodes. En développant une analyse sur un corps $K$ valué n. a. on a suivi la route qui consiste à simplement remplacer dans la théorie réelle le corps des nombres réels par $K$ et à voir ce que devient la théorie réelle. Mais cela exigeait des définitions nouvelles, adaptées au cas non-archimédien à fin d'obtenir une bonne théorie. Il y a des résultats en analyse n. a. qui sont tout à fait analoguesà des propriétés de l'analyse réelle, mais dont la démonstration diffère complètement de celles de l'analyse réelle, par exemple pour le théorème de HahnBanach. Une unification des méthodes de démonstration serait certainement désirable en tant que de beauté mathématique. Mais il me semble qu'on ne doit pas être trop optimiste pour qu'une théorie unifiée soit possible. Il faut observer qu'il n'y a pas un ordre sur le corps $\mathrm{K}$ ce qui a pour conséquence que partout où en analyse réelle on fait usage de l'ordre sur le corps des nombres réels il faut trouver des moyens en analyse n. a. pour le remplacer par des méthodes n'utilisant que la norme, 
soit la norme sur $K$, soit la norme non-archimédienne sur les espaces de Banach $n$. $a$. Il est évident que cela a des conséquences profondes en ce qui concerne les méthodes à utiliser et les résultats de la théorie. Il semble difficile, par exemple, dè généraliser une notion telle qu'une intégrale positive de l'analyse réelle. D'autre part, ces nouvelles méthodes pourraient être intéressantes pour l'analyse réelle afin de savoir quiels résultats de l'ánalyse réelle peuvent être obtenus sans usage de la notion d'ordre peut être formulés seulement en termes de la norme.

III. Résultats. Dans tout ce qui suit je désigne par $K$ un corps muni d'une valuation non-archimédienne non-triviale, c'est-à-dire la valuation vérifie l'inégalité

$$
|x+y| \leqslant \max (|x|,|y|), \quad x, y \in K
$$

Je suppose $\mathrm{K}$ complet par rapport à la métrique déduite de la valuation.

Faisons d'abord quelques remarques générales.

Je veux traiter dans ce qui suit la théorie des espaces de Banach n.a. et des espaces localement convexes sur K. Un tel programme doit être placé dans le cadre général des espaces linéaires topologiques sur K. On trouve des résultats généraux chez Bourbaki; on $\mathrm{y}$ démontre, par exemple, le théorème dè Banach, le théorème du graphe fermé, le théorème de Banach-Steinhaus.

Il y a beaucoup de résultats de l'analyse réelle qui subsistent en analyse n.a., même parmi ceux dont on ne s'en attendrait pas, par exemple la convexité et la séparation de convexes par des hyperplans, qui, en analyse réelle, sont formulés au moyen de l'ordre sur $R$.

Mais il y a aussi des différences inattendues, par exemple en ce qui concerne le réflexivité. Signalons une difficulté. Les espaces de Banach n. a. sur K, c'està-dire les espaces normés complets $E$ sur $K$ dont la norme satisfait à l'inégalité

$$
\|x+y\| \leqslant \max (\|x\|,\|y\|), \quad x, y \in E,
$$

ont la dimension 0 . C'est le même pour les espaces localement convexes sur $\mathrm{K}$. La topologie n'est donc que très faible et cela présente des difficultés sérieuses pour certains problèmes. On les rencontre notamment dans la transcription des résultats de l'analyse réelle où la frontière de certains ensembles semble essentielle : dans ces cas la frontière de ces ensembles est vide en analyse non-archimédienne. 


\section{§.1. Notions géométriques préliminaires.}

J'introduirai quelques notions qui sont d'un usage permanent dans toute la théorie. Je désigne par $\mathrm{E}$ un espace linéaire sur $\mathrm{K}$.

Définition 1.1. Un ensemble $C \subset E$ est appelé convexe si $x, y, z \in C$ entraîne

$$
\lambda x+\mu y+\nu z \in C \text { pour tout } \lambda, \mu, \nu \in K,
$$

$|\lambda| \leqslant 1, \quad|\mu| \leqslant 1, \quad|\nu| \leqslant 1, \quad \lambda+\mu+\nu=1$.

Une définition équivalente est la suivante : C est convexe si cet ensemble peut être transformé par une translation en un ensemble $C^{\prime}$ satisfaisant

$\mathrm{x}, \mathrm{y} \in \mathrm{C}^{\prime} \Longrightarrow \lambda \mathrm{x}+\mu \mathrm{y} \in \mathrm{C}^{\prime}$ pour tout $\lambda, \mu \in K,|\lambda| \leqslant 1,|\mu| \leqslant 1$. Observons que cette translation $n^{\prime}$ est pas uniquement déterminée

Si $p$ est une semi-norme $n$. a. sur $E$, c'est-à-dire une application $E \rightarrow R_{+}$ satisfaisant $p(a x)=|a| p(x)$ pour tout a $\in K$ et $p(x+y) \leqslant \max (p(x), p(y))$, les p-boules

$$
\left\{x \in E \mid p\left(x-x_{0}\right) \leqslant r\right\}
$$

sont des ensembles convexes. En particulier, si E est normé n. a. les boules $\left\|x-x_{0}\right\|<r$ et $\left\|x-x_{0}\right\| \leqslant r$ sont convexes. Le centre d'une telle boule n'est pas uniquement déterminé : tout point de la boule peut servir comme centre. Les boules possèdent des propriétés curieuses : si deux boules ont une intersection non vide, une de ces boules est contenue dans l'autre.

Les ensembles convexes possèdent les propriétés élémentaires comme dans le cas réel. En particulier à tout convexe absorbant A une notion qu'on définit d'une façon évidente - on peut associer une semi-norme n. a. p et on a

$$
\{x \in E \mid p(x)<1\} \subset A \subset\{x \in E \mid p(x) \leqslant 1\}
$$

Cependant, une telle semi-norme $\mathrm{p} n$ 'est pas unique ; il existe une semi-norme maximale et une semi-norme minimale qui sont identiques si et seulement si la valuation est dense.

Signalons une situation désagréable. Lorsqu'on définit sur E une topologie au moyen de $\mathrm{p}$, les ensembles à droite et à gauche de ces inclusions sont à la fois ouverts et fermés de sorte que les frontières de ces ensembles sont vides. C'est la 
difficulté que je signalais ci-dessus. On pourrait se demander si la définition de la convexité que j'ai donnée ci-dessus est la meilleure et si l'on pourrait éviter cette difficulté en partant d'une autre définition. Mais cela ne me semble pas très probable puisque, à part les difficultés avec la frontière, on peut construire une théorie satisfaisante au moyen de la définition donnée ci-dessus.

Je viens à une autre notion d'importance fondamentale.

Soit $(E, p)$ un espace linéaire sur $K$, muni d'une semi-norme n. a. $p$.

Définition 1.2. ( $E, p)$ sera appelé $p$-sphériquement complet lorsque toute famille de p-boules qui est totalement ordonnée par inclusion a une intersection non-vide.

Il revient au même de dire que toute famille décroissante de p-boules a une intersection non-vide. Si $\mathrm{p}$ est une norme on dit que l'espace normé E est sphériquement complet. Cette définition s'applique au corps $K$, considéré somme espace sur lui-même; on dit alors que $\mathrm{K}$ est sphériquement complet.

Il faut comparer ce théorème à la propriété bien connue que parmi les espaces métriques les espaces complets sont caractérisés par le fait que chaque suite de boules décroissante dont les diamètres tendent vers 0 a une intersection non vide.

Considérons les espaces normé n. a.

(i) Chaque espace sphériquement complet est complet, mais pas inversement.

Définition. 1.3. La norme n. a. sur E est appelée discrète si dans

$$
\mathrm{N}_{E}=\{\|x\| \mid x \in E\}
$$

toute suite strictement décroissante tend vers 0 .

Evidemment l'espace $E$ ne peut satisfaire cette condition que si la valuation de $\mathrm{K}$ est discrète. Cependant, cette condition $\mathrm{n}^{\prime}$ implique pas que $\mathrm{N}_{\mathrm{E}}$ soit discret dans $\mathrm{R}_{+}^{*}$; on connaît des contre-exemples.

(ii) Chaque espace de Banach n. a. dont la norme est discrète est sphériquement complet.

(iii) Chaque corps valué discret, en particulier tout corps localement compact, est sphériquement complet.

(iv) Il existe des corps à valuation dense qui sont sphériquement complet. 
Exemple. Soit $\Gamma$ un corps. Les éléments $\neq 0$ du corps $K$ sont toutes les séries formelles

$$
x=\sum a_{\gamma}{ }^{\alpha_{r}}, a_{\gamma} \in \Gamma,
$$

telles que

$$
v_{x}=\left\{r \mid a_{r} \neq 0\right\}
$$

est une section des ordinaux,

$$
\alpha_{r} \in Q \text { et } \gamma \longmapsto \alpha_{\gamma} \text { est croissante sur } v_{x} \text {. }
$$

En définissant la valuation par

$$
|x|=2^{-\alpha r},|0|=0
$$

où $r$ est le plus petit élément de $\mathrm{V}_{\mathrm{x}}, \mathrm{K}$ est sphériquement complet.

Le sous-corps $\mathrm{L}$ de $\mathrm{K}$, dont les éléments sont les séries formelles telles que $\{\gamma \in Q \mid a r \neq 0\}$ est un ensemble fini ou un ensemble tendant vers $\infty$, n'est pas sphériquement complet.

(v) On peut caractériser les corps sphériquement complet d'une façon algébrique. On démontre que $K$ est sphériquement complet si et seulement si $K$ est maximalement complet en ce sens qu'il n'existe pas d'extension valuée de $K$ qui a le même groupe des valeurs et le même corps résiduel que $\mathrm{K}$.

Remarquons que la notion d'espace sphériquement complet a le caractère d'une sorte de compacité. La compacité au sens usuel, cependant, est parfois trop restrictive pour l'analyse non-archimédienne; elle entraîne par exemple que la valuation du corps $\mathrm{K}$ soit discrète. La notion "sphériquement complet" semble mieux adaptée à la théorie non-archimédienne.

Gruson (1966) a étudié cette notion dans le cadre de ses recherches sur les propriétés catégorielles des espaces de Banach n. a.; on y trouve des résultats sur l'ensemble des "trous" d'un espace de Banach n. a. Springer (1965) a défini la notion d'ensemble c-compact dans les espaces linéaires sur $\mathrm{K}$ qui, pour les corps, est en relation avec la notion de corps sphériquement complet. Son point de départ est la notion de filtre convexe sur $E$ ( $O u$ sur une partie $X$ de $E$ ), ce qui est un filtre qui possède une base formée de parties convexes. Alors X $C E$ est dite 
c-compact si tout filtre convexe sur $X$ possède au moins un point adhérent dans $X$. Il démontre, parmi d'autres propriétés, que $K$ est sphériquement complet si et seulement si K est c-compact. Remarquons qu'un ensemble c-compact n'est pas nécessairement borné : tout corps sphériquement complet est c-compact, cependant non borné.

Ceci sont les outils nécessaires pour la théorie des espaces de Banach n. a.

\section{§.2. Structure des espaces de Banach n. a.}

Un problème classique ouvert dans la théorie des espaces de Banach réels est le problème de l'existence d'une base (Schander-base). On peut dire que ce problème est résolu au cas non-archimédien.

Soit $E$ un espace de Banach $n$. a. sur $K$. Le problème est de donner des conditions nécessaires et suffisantes pour qu'il existe dans $E$ une suite $\left(e_{i}\right)_{i \in I}$ telle que chaque $x \in E s^{\prime e ́ c r i t ~ d ' u n e ~ f a c ̧ o n ~ u n i q u e ~ c o m m e ~ s e ́ r i e ~}$

$$
x=\sum_{i=1}^{\infty} x_{i} e_{i}, x_{i} \in k
$$

la convergence de la série doit être entendue au sens de la convergence suivant la norme. Observons que dans le cas non-archimédien une telle série converge si et seulement si $\lim _{i \rightarrow \infty} x_{i} e_{i}=0$. J'ai commencé l'étude de ce problème en 1948, conduisant à un résultat pour le cas d'espaces séparables. J'ai été guidé par une analogie avec la théorie des espaces de Hilbert en introduisant une notion d'orthogonalité. Cela doit se faire sans usage d'un produit scalaire puisque, même dans les espaces de Jimension $n \geqslant 5 \operatorname{sur} Q_{p}$, il n'existe pas un produit scalaire au sens usuel. $L^{\prime}$ orthogonalité fut donc introduit d'une manière géométrique dont je parlerai plus loin. Cela a conduit à la notion de "famille orthogonale" et les théorèmes d'existence suivants (Monna, Springer 1965).

Définition 2.1. Une famille de vecteurs $\left(e_{i}\right)_{i \in I}, e_{i} \in E, e_{i} \neq 0$, est appelée orthogonale si

$$
\left\|\sum_{i=S} \lambda_{i} e_{i}\right\|=\max _{i \in S}\left\|\lambda_{i} e_{i}\right\|
$$

pour tout $\lambda_{i} \in \mathrm{K}$ et tout ensemble fini $\mathrm{S} C \mathrm{I}$.

On a alors

$$
\left\|\sum \lambda_{i} e_{i}\right\|=\sup \left\|\lambda_{i} e_{i}\right\|
$$


pour toute série convergente $\sum \lambda_{i} e_{i}$.

Définition 2:2. Une famille orthogonale $\left(e_{i}\right)_{i \in I}$ est appelée une base orthogonale de $E$ si tout $x \in E$ s'écrit dans la forme d'une série convergente $\Sigma \lambda{ }_{i} e_{i}$; cela veut dire que $\lambda_{i} e_{i}$ tend vers 0 suivant le filtre des complémentaires des parties finies de I. On dit alors que E est orthogonalisable.

Pour toute base orthogonale on a

$$
\|x\|=\sup _{i}\left\|\lambda_{i} e_{i}\right\|
$$

On a le théorème suivant.

Théorème 2.1. Chaque espace de Banach dont la norme est discrète est orthogonalisable.

On peut démontrer ce théorème en construisant une base algébrique pour

$$
E_{\lambda}=\{x \in E \mid\|x\| \leqslant \lambda\}, \lambda>0,
$$

qui est un module sur l'anneau des entiers de $K$, et en élevant cette base à une base pour E ; j'omets les détails de la démonstration, mais on voit que c'est une méthode adaptée au cas non-archimédien et qu'on ne peut pas utiliser en analyse réelle.

Ce théorème permet de donner une caractérisation d'une classe d'espaces de Banach n. a. D'abord un exemple.

Soit I un ensemble et soit $c(I) I$ 'ensemble des familles $x=\left(x_{i}\right)_{i \in I}, x_{i} \in K$, telles que $x_{i}$ tende vers 0 suivant le filtre des complémentaires des parties finies de I. En posant $\|x\|=\sup _{i} x_{i}, c(I)$ est un espace de Banach n. a. 
Posons $N_{K}=\{|a| \mid a \in K\}$. Ici se présente une situation qui n'a pas d'analogue en.analyse réelle. En analyse n. a. $\mathrm{N}_{E}$ n'est pas nécessairement une partie de $\mathrm{N}_{\mathrm{K}}$; on peut sans peine construire des exemples.

Supposons $\mathrm{N}_{\mathrm{E}} \subset \mathrm{N}_{\mathrm{K}}$. Pour que la norme soit discrète il faut et il suffit alors que la valuation de $\mathrm{K}$ soit discrète. Dans ce cas on peut arranger que la norme de chaque vecteur de la base est égale à 1 ; on obtient alors une base orthogonale.

Corollaire 2.2. Supposons que la valuation de K soit discrète. Tout espace de Banach n. a. vérifiant $N_{E} \subset N_{K}$ est isomorphe avec sa norme à un espace $c(I)$.

Si $N_{E}$ n'est pas une partie de $N_{K}$, on peut obtenir le résultat suivant par une renormalisation.

Corollaire 2.3. Supposons que la valuation de K soit discrète. Tout espace de Banach n. a. est isomorphe comme espace vectoriel topologique à un espace c(I).

Serre a montré qu'on peut affaiblir la condition $\mathrm{N}_{E} \subset \mathrm{N}_{\mathrm{K}}$.

Dans le cas suivant on peut encore démontrer l'existence d'une base orthogonale. On dit que l'espace $E$ est de type dénombrable, lorsqu'il contient un sousespace dense de dimension finie ou dénombrable.

Théorème 2.4. Supposons que $K$ soit sphériquement complet (c-compact). Alors tout espace de Banach n. a. sur $K$ de type dénombrable est orthogonalisable.

La démonstration peut se faire par une procédé d'orthogonalisation, comparable au procédé de Gram-Schmidt.

Il existe des espaces qui ne sont pas orthogonalisables.

Désignons par $l^{\infty} I^{\prime}$ espace des suites bornées $\left(x_{i}\right)_{i} \in I$ normé par $\sup \left|x_{i}\right|$. On démontre sans peine que $\ell^{\infty}$ est sphériquement complet si $\mathrm{K}$ est c-compact.

Théorème 2.5. Soit $\mathrm{E}$ un espace de Banach $\mathrm{n}$. a. orthogonalisable de dimension infinie dont la norme n'est pas discrète. Alors $E$ n'est pas sphériquement complet.

Théorème 2.6. Soit $\dot{K}$ un corps c-compact à valuation non discrète. Alors $l^{\infty} \underline{n^{\prime} e s t}$ pas orthogonalisable.

Carpentier (1965) a analy,sé en détail le problème de l'existence de bases orthogonales et il a montré que les résultats que j'ai donnés ci-dessus sont les meilleurs possibles. 
Mentionnons encore le résultat suivant, qui est une généralisation d'un théorème bien connu sur les espaces de Banach localement compacts réels.

Théorème 2.7. Soit $\mathrm{E}$ un espace normé $\mathrm{n}$. a. sur $\mathrm{K}$ qui est lecalement c-compact.

Alors $K$ est $c$-compact et la dimension de $E$ est finie.

Pour la démonstration on utilise les familles orthogonales. Gruson a montré que chaque sous-espace fermé d'un espace de Banach n. a. orthogonalisable est luimêne orthogonalisable.

Van der Put a introduit la notion de famille $\alpha$-orthogonale qui s'est montrée utile. Etant donné $\alpha, 0<\alpha \leq 1$, une famille $\left(e_{i}\right)_{i \in I}, e_{i} \in E$, est appelée $\alpha$-orthogonale si

$$
\left\|\sum_{i \in I} \lambda_{i} e_{i}\right\| \geqslant \alpha \sup _{i \in I}\left\|\lambda_{i} e_{i}\right\|
$$

pour toute série convergente $\sum \lambda_{i} e_{i}$. La notion de $\alpha$-base s'introduit d'une façon évidente. Van der Put a démontré le théorème suivant.

Théorème 2.8. (i) Soit $E$ un espace normé n. a. de type dénombrable. Alors $E$ possède une $\alpha$-base dénombrable ou finie pour tout $\alpha, 0<\alpha<1$.

(ii) Soit $E$ un espace normé $n$. a. sur un corps $K$ a valuation discrète. Alors $E$. possède une $\alpha$-base pour tout $\alpha, 0<\alpha<1$.

Voir aussi un travail de Bosch sur l'existence de bases.

Les bases orthogonales s'appliquent dans diverses domaines de l'analyse nonarchimédienne. Je rappelle les travaux de Mme Amice sur l'interpolation p-adique, conduisant à une base pour certaines algèbres de fonctions continues. Le théorème de Van der Put sur les équations différentielles que j'ai mentionné au début fut démontré par lui avec l'aide des bases orthogonales. Enfin je rappelle la théorie des fonctions analytiques non-archimédiennes et la théorie des algèbres affinoides (Grauert, Remmert).

J'ai déjà dit que la dénomination base orthogonale trouvait son origine dans une notion d'orthogonalité. Puisqu'une telle notion ne peut s'introduire au moyen d'un produit scalaire, j'ai choisi une autre voie, à savoir la notion de "plus petite distance", ce qui, dans l'espace de Hilbert, en est équivalente. C'est donc liée à la notion de "meilleure approximation". 
Soit $E$ un espace de Banach $n$. a., et soient $x, y \in E$. On dit que $x$ est orthogonal à̀ y si

$$
\inf _{\lambda \in K}\|x-\lambda y\|=\|x\| \text {, }
$$

qui signifie que $\|x\|$ est la plus petite distance de $x$ à l'espace linéaire engendré par y.

Plus généralement on dit que $x$ est orthogonal à un sous-espaces linéaire fermé $V$ de $E$ si $\|x\|$ est la plus petite distance de $x$ à V. Il faut se garder. Si $x \notin V$, la plus petite distance d de $x$ à $V$ est $\neq 0$, mais il se peut qu'il n'existe pas a $\in V$ tel que $d=\|x-a\|$. On démontre sans peine qu'il en existe si $V$ est sphériquement complèt. Mais dans ce cas un tel point a - la projection de $x$ sur V n'est pas uniquement déterminé. On a les propriétés suivantes :

(i) Si $\mathrm{x}$ est orthogonal à $\mathrm{y}, \mathrm{y}$ est orthogonal à $\mathrm{x}$.

(ii) $x$ est orthogonal à y si et seulement si

$$
\|\alpha x+\beta y\|=\max (\|\alpha x\|,\|\beta y\|)
$$

pour tout $\alpha, \beta \in K$.

C'est cette dernière propriété qui est à la base de la théorie des familles orthogonales. Voir un travail de Treiber [21].

On a pu construire une théorie d'orthogonalité : une notion de supplémentaire orthogonal d'un sous-espace linéaire (ce qui, cependant, s'il en existe, n'est pas unique); une théorie des projecteurs qui préseñte certaines analogies avec la théorie des projecteurs dans l'espace de Hilbert; une correspondance entre les projecteurs et les supplémentaires orthogonaux. Il me semble que le rôle des projecteurs dans la théorie des opérations linéaires pourrait être un sujet de recherche (décompositions spectrales).

Récemment Mme. De Grande-De Kimpe a étudié la relation entre cette notion d'orthogonalité et la théorie de la meilleure approximation. Elle a commencé l'étude des bases dans les espaces localement convexes n. a. [8].

On a étudié la dualité dans les espaces de Banach $n$. a. - ce qui a conduit à des différences remarquables avec la théorie des espaces réels - mais il me semble mieux de rapporter sur ces résultats dans le cadre du théorème de Hahn-Banach et ses conséquences. 


\section{§.3. Le théorème de Hahn-Banach.}

Dans ce paragraphe il s'agit de la théorie des fonctionnelles linéaires continues, et plus généralement des'applications linéaires, dans les espaces nonarchimédiens.

D'abord on étudiait ces problèmes dans les espaces de Banach n. a.; en 1943 j'ai donné une condition suffisante pour l'existence de fonctionnelles linéaires continues $\neq 0$ (à savoir la condition $N_{E}$ soit discrèt en $R_{+}^{*}$ ); Cohen (1948) a montré qu'il suffit que la valuation de K soit discrète. Enfin Ingleton (1952) a donné une condition nécessaire et suffisante, toujours dans les espaces normés. En 1958 j'ai généralisé ce résultat pour les espaces localement convexes.

Observons que, dès qu'on possède la notion d'ensemble convexe, les espaces localement convexes sur $K$ sont définis comme dans le cas réel, soit de façon géométrique, soit par une famille de semi-normes $\mathrm{n}$. a. Le théorème de Kolmogoroff donnant un critère pour que la topologie se déduise d'une norme subsiste au cas n.a. Remarquons que les fonctionnelles linéaires prennent par définition même leurs valeurs dans le corps $\mathrm{K}$. La relation entre la continuité et les applications bornées subsiste ; la démonstration doit être adaptée au cas non-archimédien. Je peux supprimer les détails de ceci.

Je viens au théorème fondamental. Soient $\mathrm{E}$ et $\mathrm{F}$ des espaces linéaires sur $\mathrm{K}$. Soit $\mathrm{p}$ une semi-norme $n$. a. sur $E$ et $q$ une semi-norme $n$. a. sur F. Soit $V$ un sousespace linéaire de $\mathrm{E}$. Soit $\mathrm{T}$ une application linéaire bornée de $\mathrm{V}$ dans $\mathrm{F}$; cela veut dire que

$$
\begin{aligned}
& \|T\|=\sup _{p(x) \neq 0} \frac{q(T(x))}{p(x)}<\infty, \\
& q(T(y)) \leqslant\|T\| \cdot p(y), y \in V .
\end{aligned}
$$

Théorème 3.1. Supposons que F. soit q-sphériquement complet. Alors il existe une application linéaire bornée $T_{0}$ de $E$ dans $F$ qui est un prolongement de $T$ tel que $\|\mathrm{T}\|=\left\|\mathrm{T}_{0}\right\|$.

Ce théorème s'applique évidement aux espaces localement convexes sur $\mathrm{K}$. En prenant pour $F$ le corps $K$, considéré comme espace sur lui-même, on obtient un théorème du type du théorème de Hahn-Banach classique. Je remarque que la démonstration usuelle du théorème de Hahn-Banach au cas réel, utilisant l'ordre sur $\mathbb{R}$, 
ne s'applique pas dans le cas non-archimédien.

Le théorème donne une condition suffisante. Mais la condition est aussi nécessaire, comme on le voit du théorème suivant, dû également à Ingleton dans le cas d'espaces normés.

Théorème 3.2. Soit q une semi-norme n. a. sur $F$ et supposons que $F$ ne soit pas q-sphériquement complet. Alors il existe un espace linéaire $E$ qui ne possède pas la propriété que chaque application linéaire bornée d'un sous-espace linéaire $\mathrm{V}$ de $\mathrm{E}$ dans $\mathrm{F}$ admet un prolongement linéaire de la même norme de $\mathrm{E}$ tout entier dans $\mathrm{F}$.

D'après ces théorèmes une condition nécessaire et suffisante pour que le théorème de Hahn-Banach soit vrai pour tout espace de Banach $n$. a. est que $K$ soit c-compact. Pour les espaces normés $\mathrm{n}$. a. de type dénombrable sur un corps $\mathrm{K}$ quelconque muni d'une valuation $\mathrm{n}$, $\mathrm{a}$, on peut encore démontrer un théorème de prolongement lorsque on fait tomber la restriction de l'invariance de la norme de l'application.

Le théorème a des conséquences évidentes sur l'existence de formes linéaires continues $\neq 0$ sur un espace $n$. a. si $K$ est c-compact; je peux les supprimer. En se rappelant qu'il existe des espaces vectoriels topologiques sur $\mathbb{R}$, non des espaces de Banach, dont le dual se réduit à 0 , le résultat suivant est remarquable :

Pour tout corps qui n'est pas c-compact, il existe un espace normé $n$. a. (non réduit à 0 ) sur $K$ dont le dual est 0 .

Exemple. Soit $K$ un corps non c-compact. Soit $E$ une extension immédiate maximale de $\mathrm{K}$ et considérons $\mathrm{E}$ comme espace sur $\mathrm{K}$. On démontre que toute forme linéaire continue sur $E$ est 0 .

En vue des théorèmes structurels du paragraphe précédent la détermination du dual d'un espace orthogonalisable vérifiant $N_{E} \subset N_{K}$ est facile : le dual est isomorphe à un espace formé de suites bornées.

Les théorèmes précédents sont très remarquables. On sait que dans le cas réel le prolongement d'applications linéaires dans un espace réel $F \neq \mathbb{R}$ avec invariance de la norme est en général impossible. Nachbin (1950) a donné une condition nécessaire et suffisante, à savoir une condition qu'il a appelée "binary intersection. property". Dans le cas non-archimédien cette condition se réduit à la notion d'espace sphériquement complet en vertu des propriétés des boules. La démonstration de Nachbin, n'utilisant pas l'ordre, fournit donc l'unification des théories dont j'ai parlée au début autant qu'il s'agit du théorème de Hahn-Banach. 
C'est ainsi que dans le cas non-archimédien on obtient une théorie valable dans des situations beaucoup plus générales que dans le cas réel. On pourrait s'attendre que cette situation conduise à des applications en analyse non-archimidienne. Cependant, elles ne me sont pas connues.

Tout ceci conduit à la théorie de la dualité pour les espaces de Banach et les espaces localement convexes $n$. a. sur un corps valué n. a. Il y a des résultats remarquables tout différents de ceux de la théorie réelle.

J'ai abordé l'étude de la dualité en 1948 pour les espaces normés. L'étude fut continué par Van Tiel et Van der Put (1969) dans un nombre d'articles. Je donne quelques résultats.

i) Tout espace de Banach réflexif sur un corps c-compact est de dimension finie.

La démonstration se fait avec la théorie des familles orthogonales. Si la valuation de $K$ est discrète et si $N_{E} C N_{K}$ cette propriété résulte d'ailleurs facilement par une comparaison des cardinaux de la base orthogonale de $E$ et de l'espace dual de $E$, qui est aussi orthogonalisable.

La situation est différente si le corps $\mathrm{K}$ n'est pas c-compact. Désignons par $c_{0} l^{\prime}$ espace de Banach des suites $\left(x_{i}\right)$, convergentes vers 0 , normé par sup $\left|x_{i}\right|$ et par $l^{\infty}$ l'espace des suites bornées, normé par la même norme. Alors on démontre :

ii) Si K n'est pas c-compact, $c_{0}$ et $l^{\infty}$ sont réflexifs.

On démontre que pour chaque $f \in\left(l^{\infty}\right)$ ' il existe $a=\left(a_{1}, a_{2}, \ldots\right) \in c_{0}$ tel que $f(x)=\sum a_{n} x_{n}$ pour tout $x=\left(x_{n}\right) \in l^{\infty}$ et que $\|f\|=\|a\|$.

Récemment Van der Put a repris l'étude de la théorie de la réflexivité, maintenant pour les corps non c-compacts. Parmi ses résultats mentionnons qu'il existe des espaces de Banach $n$. a. sur un corps non c-compact qui sont isomorphes à leur dual. Un exemple d'un tel espace est l'espace $c_{0} \oplus l^{\infty}$, muni de la norme n. a. $\max (\|x\|,\|y\|), x \in c_{0}, y \in l^{\infty}$. Il a posé la conjecture suivante :

Conjecture : Supposons $\mathrm{K}$ non c-compact. Si le cardinal de $\mathrm{E}$ est non-mesurable, le dual de $E$ est réflexif.

Il supporte cette conjecture par un nombre d'exemples. 
Van Tiel a étudié la dualité pour les espaces localement convexes n. a. Il a démontré certains critères généraux. Je remarque que la situation diffère essentiellement du cas des espaces normés. Il démontre, en donnant des exemples, que pour tout corps c-compact il existe des espaces localement convexes réflexifs de dimension infinie.

Mentionnons enfin le résultat suivant, tout différent de la situation en analyse réelle.

Supposons K c-compact ; soit $E$ un espace de Banach n. a. sur $K$ vérifiant $N_{E} \subset N_{K}:$ Alors toute suite $\left(x_{n}\right)$ faiblement convergente vers $x$, converge fortement vers $x$.

\section{\$.4. Structure géométrique.}

En analyse réelle on connaît une forme géométrique du théorème de Hahn-Banach équivalente à la forme analytique que j'ai traitée au $\$ .3$. En analyse non-archimédienne il existe aussi une forme géométrique, mais alors quelques précautions, qui sont caractéristiques pour ce cas, sont nécessaires.

Supposons que $\mathrm{K}$ soit c-compact. Soient A C E un ensemble convexe absorbant et $\mathrm{p}$ une semi-norme n. a. telle que

$$
\{x \in E \mid p(x)<1\} \subset A \subset\{x \in E \mid p(x) \leq 1\} .
$$

Posons

$$
A_{p}=\{x \in E \mid p(x)<1\}
$$

On a alors le théorème suivant.

Théorème 4.1. Soit $V$ une sous-variété affine dans $E$ telle que $\AA_{P} \cap V=\varnothing$. Alors il existe dans $E$ un hyperplan affine $W$ tel que $V \subset W$ et $\AA_{p} \cap W=\varnothing$.

Ce théorème est équivalent à la forme analytique du théorème de Hahn-Banach.

En analyse réelle on exprime ce théorème pour les ensembles convexes ouverts mais, à cause des difficultés topologiques dont j'ai parlées dans l'introduction, cela ne peut se faire dans le cas non-archimédien. Les ensembles à droite et à gauche dans ces inclusions sont à la fois ouverts et fermés. C'est pour cela qu'il 
faut exprimer le théorème par rapport à $A_{p}$.

Ce théorème est le point de départ pour des théorèmes concernant la séparation de convexes par des hyperplans. En analyse réelle cela se fait par la notion de "demi-espaces" déterminés par un hyperplan, par exemple $\{x \in E \mid F(x)>0\}$ resp. $\{x \in E \mid F(x)<0\}$. En absence d'un ordre sur $K$ une telle notion ne peut être définie de la façon usuelle en analyse non-archimédienne. Il sera donc nécessaire de suivre une autre voie pour définir ce qu'il faut entendre par une proposition telle que "les points $\mathrm{x}$ et $\mathrm{y}$ de $\mathrm{E}$ sont situés d'un même côté d'un hyperplan $H^{\prime}$. Il est remarquable que cela peut se faire malgré l'absence d'un ordre et qu'on peut donner une théorie de la séparation semblable à la théorie réelle.

Soit $E$ un espace Iinéaire sur K. Désignons par $c(\{x, y\})$ I'enveloppe convexe de l'ensemble $\{x, y\} ;$ il se compose des points $z=\lambda x+(1-\lambda) y,|\lambda| \leqslant 1$. Soit $F$ une forme linéaire sur E.

Désignons par H l'hyperplan défini par l'équation $F(x)=\alpha(\alpha \epsilon K)$. J'ai donné la définition suivante (1964) :

Définition 4.1.

(i) $\mathrm{x}, \mathrm{y} \in E(\mathrm{x} \notin \mathrm{H}, \mathrm{y} \notin \mathrm{H})$ sont dits séparés par $\mathrm{H}$ si

$$
\mathrm{C}(\{\mathrm{x}, \mathrm{y}\}) \cap \mathrm{H} \neq \varnothing \text {. }
$$

(ii) On dit que $\mathrm{x}, \mathrm{y} \in \mathrm{E}(\mathrm{x} \notin \mathrm{H}, \mathrm{y} \in \mathrm{H})$ sont situés d'un même côté de $\mathrm{H}$ si

$$
C(\{x, y\}) \cap H=\varnothing
$$

(iii) On note $\mathrm{x} \sim \mathrm{y}$ si $\mathrm{x}$ et $\mathrm{y}$ sont situés d'un même côté de $H$. On voit sans peine que la relation $\sim$ est une relation d'équivalence. Les classe d'équivalences sont appelées les côtés de H.

La même méthode peut s'appliquer en analyse réelle et conduit alors à la situation usuelle, c'est-à-dire on obtient les deux côtés (demi-espaces) usuels. Mais en analyse non-archimédienne tout hyperplan a une inifinité de côtés (pourvu que la valuation de $K$ ne soit pas triviale), dont le cardinal ne dépend que de $K$. Les côtés sont convexes et, si $E$ est un espace vectoriel topologique sur $K$, ils sont à Ia fois ouverts et fermés. On peut alors construire une théorie concernant la séparation d'ensembles convexes par des hyperplans; c'est une séparation stricte. 
Nous supprimons les détails de la théorie, que Van Tiel a appliquée à la théorie de la dualité. Il faut cependant remarquer qu'il y a des difficultés si on veut définir une notion d'hyperplan d'appui par la même raison que j'ai indiquée pour la forme géométrique du théorème de Hahn-Banach. J'ai essayé de surmonter ces difficultés par la définition d'une frontière par des moyens non-topologiques, mais cela n'a pas donné une théorie satisfaisante. En tout cas cette voie n'a pas conduit à un théorème analogue au théorème de Krein-Milman.

Observons que la circonstance qu'un hyperplan a une infinité de côtés a été utilisée par Carpentier pour démontrer des théorèmes concernant la séparation de plusieurs convexes par des hyperplans ; évidemment cela n'a pas d'analogue en analyse réelle. Il a aussi donné une caractérisation des ensembles convexes compacts dans le cas d'une valuation discrète de $\mathrm{K}$. Il démontre que si la dimension de $\mathrm{E}$ est infinie, les points d'un convexe compact peuvent être exprimés sous la forme d'une série

$$
x=\sum_{i=1}^{\infty} \lambda_{i} e_{i}, e_{i} \in E, \lambda_{i} \in \mathrm{K},
$$

où $\left|\lambda_{i}\right| \leqslant \alpha_{i}$ et la suite $\left(\alpha_{i}\right)$ tend vers 0 . Si la dimension de E est finie, on obtient évidemment une série finie.

L'application de la théorie de la séparation au corps $\mathrm{K}$ lui-même, considéré comme espace vectoriel sur lui-même, par rapport à la fonction $\mathrm{x} \rightarrow \mathrm{ax}, \mathrm{a} \epsilon \mathrm{K}^{*}$, conduit à une notion de pseudo-ordre sur un corps valué n. a., qui remplace l'ordre usuel sur $\mathbb{R}$. On obtient alors une infinité de côtés de o comme classes d'équivalences, qui constituent une partition de $\mathrm{K}$. Les côtés forment un groupe abélien infini G lorsqu'on définit la multiplication de deux côtés $\dot{S}_{1}$ et $S_{2}$ par

$$
s_{1} S_{2}=\left\{x y \mid x, y \in K^{*}, x \in s_{1}, y \in s_{2}\right\}
$$

ce qui est justifié puisqu'on démontre que cet ensemble est encore un côté de 0 . Ce groupe exprime une généralisation des règles pour la multiplication des nombres réels positifs et négatifs. Par analogie à la théorie dans $\mathbf{R}$ on définit alors un pseudo-ordre $G$ sur $K$ de la façon suivante :

Définition 4.2. Soit $S \in G$. On écrit $a \geqslant s^{0} \underline{\text { si }} a=0$ ou a $\in S$. On écrit $a \geqslant s^{b} \underline{\text { si }}$ et seulement $s i a-b \geqslant s^{0}$. 
Un pseudo-ordre $G$ est donc déterminé par une infinité de relations.

Observons que la même méthode, appliquée au corps $\mathbf{R}$ des nombres réels, considéré comme corps valué, conduit à l'ordre usuel sur $\mathbb{R}$. Sur le corps $\mathbf{Q}$ des nombres rationnels on peut définir soit (i) l'ordre usuel, soit (ii) un pseudo-ordre lorsqu'on considère $Q$ comme corps p-adique.

Pour le pseudo-ordre sur $\mathrm{K}$ on démontre des règles de calcul, en une certaine mesure analoguesaux axiomes pour les corps ordonnés : Supposons que la valuation de $\mathrm{K}$ est telle que $|2|=1$. On a les règles :

(a) Pour tout $a \neq 0$ il existe un côté unique $S \in G$ tel que a $>{ }_{S}{ }^{0}$.

(b) $a \geqslant S^{0}$ et $-a \geqslant S^{0} \Rightarrow a=0$.

(c) $a \geqslant s_{1} 0, b \geqslant s_{2} 0 \Longrightarrow a b \geqslant s_{1} s_{2} 0$.

(a) Soient $a \geqslant S_{1} 0, b \geqslant S_{2} 0$; alors il existe $S^{\prime} \epsilon G$ tel que $a+b \geqslant S^{\prime}$. 0 .

(e) $a \geqslant s^{b} \Longrightarrow a+c \geqslant s^{b+c}$ pour tout $c \in K$

(f) $a \geqslant s$ et $b \geqslant s^{a} \Longrightarrow a=b$.

Cependant la propriété transitive est en défaut ; elle est remplacée par une propriété qui découle de la convexité des côtés de 0 .

( 8 ) $a \geqslant s^{b}$ et $b \geqslant S^{c} \Longrightarrow a \geqslant 2 s^{c}$

Pour une classe de corps topologiques, non nécessairement valués, on peut définir une notion de pseudo-ordre d'une façon axiomatique. On le définit au moyen d'une partition de $K^{*}$, vérifiant certaines propriétés, généralisant les propriétés des côtés déduits d'une valuation. Pour un tel pseudo-ordre on peut, par exemple, définir une notion telle que "ensemble borné par rapport au pseudo-ordre". La théorie reste à développer. Il serait intéressant de savoir, par exemple, si les notions de "borne supérieure" et "borne inférieure" d'un ensemble "pseudo-borné" peuvent être généralisées ; cela semble difficile. Quant au pseudo-ordre j'ai posé un nombre de problèmes de caractère algébrique et topologique [12]; je ne. les répète pas ici. M. Moh. Akkar s'est occupé de ces problèmes. 
K écant un corps muni d'un pseudo-ordre G, on peut définir la notion d"espace vestoriel pseudo-ordonné sur $K^{\prime \prime}$. Cela se fait au moyen d'une famille d'ensembles ccnvexes disjoints, vérifiant des propriétés qui expriment une certaine compatibilité avec lé groupe G. Ces ensembles, en nombre infini, pr nnent la place du cône des éléments positifs de la théorie des espaces vectoriels ordonnés sur R. Une telle théorie, dont j'ai donné les définitions préliminaires, reste encore à développer. Je pose les problèmes suivants :

(i) Est-il possible de généraliser la notion de "espace de Riesz" pour les espaces pseudo-ordonnés?

(ii) Généraliser la notion de "fonctionnelle positive" pour les espaces pseudoordonnés ; récemment j'ai donné quelques suggestions.

\section{$\S .5$. Recherches variées.}

Il y a d'autres domaines de recherche en analyse non-archimédienne qui. sont liés à la théorie générale dont j'ai donné une revue dans les paragraphes précédents. Ce sont soit des applications, soit des théories générales. Je ne peux que les mentionner.

Des espaces spéciaux furent l'objet de diverses études :

(i) Espace des fonctions. entières sur un corps valué n. a. (Raghunatan).

(ii) Fonctions presque périodiques n. a. (Rangan, Van Rooy).

(iii) Espaces de Köthe (Dorleyn, De Grande-De Kimpe, Monna)

Un domaine important est la théorie de l'intégration de fonctions à valeurs dans $\mathrm{K}$, l'intégrale prenant ses valeurs dans $\mathrm{K}$. La théorie fut d'abord développé par Monna et Springer et achevée et améliorée par Van Rooy et Schikof. Mentionnons le fait remarquable qu'il existe un ensemble maximal négligeable. Le théorème de Radon-Nikodym est en défaut. Monna et Springer ont donné une condition nécessaire et.suffisante pour l'existence d'une mesure de Haar sur un groupe localement compact à valeurs dans $\mathrm{K}$. 
Ces résultats ont amené Van Rooy et Schikhof à étudier l'analyse harmonique non-archimedienné ; ils ont obtenu beaucoup, de résultats importants dans ce domaine et sur les algèbres de Banach n. a. Les algèbres n. a. furent 1'objet de recherches intéressantes de Van der Put. Dans ce domaine on rencontre des difficultés puisque le théorème de Gelfand-Mazur est en défaut au cas n. a. Mentionnons l'étude des aigèbres de Tate (Grauert, Remmert).

Dès' 'le début du développement de l'analyse n. a. on a étudié la théorie des opérations linéaires dans les espaces de Banach n. a. (Monna). Mentionnons un travail important de Serre sur les applications linéaires complètement continues d'espaces de Banach n. a. Cette étude fut continée par Grusọ dans un cas plus général. Les opérations linéaires furent aussi étudiées par Ellis, Van der Put et Van Tiel.

Comme application mentionnons une étude du problème des moments en analyse n. a. (Monna).

IV. Développement ultérieur.

Un développement ultérieur semble possible sur plusieurs domaines. J'indique quelques directions de recherches.

1 - J'ai déjà signalé les difficultés qu'on rencontre en tous les problèmes dans lesquels la frontière d'un ensemble semble essentiel. Je pense à des notions comme convexité stricte, convexité uniforme, points extrémaux, théorème de Krein-Milman. On ne sait pas de quelle façon il faut attaquer ces problèmes en analyse non-archimédienne. La topologie n'étant que très faible, il se peut qu'il serait utile d'employer des méthodes plutôt algébriques pour surmonter ces difficultés.

2 - Ces derniers temps il y a en analyse réelle des publications sur dos espaces non-localement convexes. Il en existe aussi en analyse non-archimédienne. Par exemple l'espace des suite $\left(x_{i}\right), x_{i} \in K$, telles que $\sum\left|x_{i}\right|<\infty$, normé par $\|x\|=\sum\left|x_{i}\right|$. Cette norme n'est pas non-archimédienne. Ce domaine est entièrement ouvert.

3 - J'ai déjà signalé les espaces psudo-ordonnés. Ce domaine peut être intéressant par ses relations avec la théorie de l'intégration (analogie des intégrales 
positives).

4 - La notion d'espace sphériquement complet mérite d'être étudiée d'une façon plus approfondie. On connaît des conditions suffisantes pour qu'un espace soit sphériquement complet (par exemple norme discrète) et on sait qu'il existe des espaces non-sphériquement complets. Pour les espaces ceci est presque tout ce qu'on sait. Pour les corps on a la caractérisation algébrique; mais pour les espaces c'est un domaine inconnu. D'ailleurs, c'est plutôt un problème topologique puisqu'on peut définir cette notion dans les espaces métriques.

Ceci sont des problèmes d'un caractère général. On peut évidemment ajouter des problèmes d'un caractère plus spécial. Cependant il me semble que, pour avancer L'analyse non-archimédienne, il ne faut pas sivre la voie de traduire chaque théorie et chaque propriété de l'analyse réelle en analyse non-archimédienne, bien que cela peut être utile pour avoir en réservedes exemples pour la théorie générale. Ce qu'il faut c'est une direction générale pour la recherche ultérieure. En tant qu'il s'agit de la théorie des espaces de Banach et des espaces localement convexes non-archimédiens des applications seraient désirables. Cela pourrait être des abolications dans des autres domaines de la mathématique, mais aussi en analyse nonarchimédienre elle-même. Je pense, par exemple, à la théorie des distributions, un domaine important d'application de la théorie des espaces localement convexes réels. Une théorie analogue en analyse non-archimédienne est-elle possible ? Comme une application de la théorie de l'intégration non-archimédienne je pense à une théorie de la probabilité non-archimédienne. Cela exige une théorie de la mesure, dont se sont occupés Schikhof et Van Rooy. Sans doute l'absence d'un ordre sur $K$, présentera des difficultés.

Dans un tout autre domaine on peut se demander si une application en physique aurait un sens. Les transformations de Lorentz furent l'objet de quelques études (Everett en Ulam, Beltrametti). Des considérations générales sur ce sujet furent publiés par Van der Blij et le rapporteur, conduisant à la conclusion que, à ce moment, une application en physique n'offre que peu de perspectives. 
BIBLIOGRAPHIE

[1] MONNA, A.F.

[2] BELTRAMETTI, E.G.

[3] ELLIS, R.L.

[4] DE GRANDE-DE KIMPE, N.

[5] DE GRANDE-DE KIMPE, N.

[6] DE GRANDE-DE KIMPE, N.

[7] DE GRANDE-DE KIMPE, N.

[8] DE GRANDE-DE KIMPE, N.

[9] KOHEN, A.W.

[10] MADLENER, K.E.
Analyse non-archimédienne, Springer-Verlag, Berlin 1970.

Note on the p-adic generalization of Lorentz transformations. Discrete Math., vol. 1, 239-246 (1971).

Extensions of continuous functions into locally convex spaces over non-archimedean fields, Colloquium Math. XXII, 249-250 (1971).

-compactness in locally K-convex spaces, Proc. Kon. Ned. Akad.v. Wetensch. A 74, 176-180 (1971).

Perfect locally. K-convex sequence spaces, Proc.Kon. Ned.Akad.v. Wetensch. 74, 471-482 (1971).

On the Grothendieck approximation property in nonarchimedean analysis, Nieuw Archief v. Wiskunde (3'), $x x, 242-245$ (1972).

On spaces of operators between locally $\mathrm{K}$-convex spaces, Proc.Kon.Ned.Akad.v. Wetensch. 75, 113-129 (1972).

n the structure of locally K-convex spaces with a Schauder basis, Proc. Kon.Ned.Akad.v. Wetensch. A 75, 396-406 (1972).

Analyse harmonique des opérateurs bornés dans les espaces de Banach non-archimédiens, thèse Université de Grenoble 1971.

Präkompakte und strickt-singuläre Operatoren auf Vektarräumer über nicht-archimedisch bewerteten 
Körper, Dissertation Mainz 1970.

[11] MONNA, A.F.

Quelques problèmes en analyse non-archimédienne, Nieuw Archief voor Wiskunde, (3), 19, 65-67 (1971).

[12] MONNA, A.F. Ordre, pseudo-ordre, primes et convexité, Proc. Kon.Ned.Akad.v. Wetensch. A 74, 181-190 (1971).

[13] van der PUT, M. Non-archimedean function algebras, Proc. Kon. Ned. Akad.v. Wetensch. A 74, 60-77 (1971).

[14] van der PUT, M. Difference equations over p-adic fields, Math. Ann. 198, 189-203 (1972).

[15] RAGHUNATAN, T.T. On proper bases of the space of entire functions over non-archimedean fields, Indian J. of Math. 12, 43-52 (1970).

[16] ROOY, A.C.M. Non-archimedean analysis Nieuw Archief voor Wiskunde van and SCHIKHOF, W.H. Ser. 3, XIX, 120-160 (1971).

[17] SCHIKHOF, W.H. A Radon-Nikodym theorem for non-archimedean integrals and absolutely continuous measures on groups, Proc. Kon.Ned.Akad.v. Wetensch. A 74, 78-85 (i971).

[18] SCHIKHOF, W.H Non-archimedean representations of compact groups, Compositio Math. 23, 215-232 (1971).

[19] SOMASUNDARAM, D. Inclusion Theorems on Matrix transformations of some sequence spaces over Non-archimedean Fields, Boll. Un.Mat.Ital., IV ser. 3, 953-960 (1970).

[20] SRINIVASAN, V.K. On certain summation processes in the p-adic fields, Proc.Kon.Ned.Akad.v. Wetensch. A 68, 319-325 (1965).

[21] TREIBER, D. Beiträge zur nicht-archimedischen Funktional analysis, Dissertation Köln 1971. 
A.F. MONNA

Mathematisch Instituat der

Rijksuniversiteit, Utrecht

Budapestlann 6, De Uithof

Utrecht. 